\title{
Infections through blood from heart-lung machine
}

\author{
W. CH.P. GELDOF and A. G. B R O M \\ Department of Thoracic Surgery, University Hospital, Leiden, The Netherlands
}

\begin{abstract}
Of 657 cultures of blood from the heart-lung machine during operations performed with extracorporeal circulation, $16(2 \cdot 4 \%)$ proved to be positive. Of these 16 patients, 12 showed no clinical infective symptoms. The case histories of the remaining four patients are presented. In three of these cases a plausible explanation for the infected perfusion blood was found: an infected aortic aneurysm, an infected aortic valve prosthesis, and mediastinitis. In only one case was the cause of the infection sought in the heart-lung machine.
\end{abstract}

\section{MATERIAL AND METHODS}

In an effort to establish whether the cause of postoperative infection in patients who had undergone surgery with extracorporeal circulation was to be found in the heart-lung machine the following study was carried out.

In nearly all patients treated surgically with extracorporeal circulation over a period of four years-1 April 1967 to 1 April 1971-cultures were made of blood taken from the heart-lung machine immediately after the completion of perfusion. For this purpose a needle was fixed to the arterial line and blood was collected in a sterile flask. This flask was kept in the refrigerator at $+4^{\circ} \mathrm{C}$ for a period ranging from 0 hour to a few days, whereupon the blood was cultured. The blood cultures were continued for at least one week. The tubing of the heart-lung machine was sterilized with ethylene oxide. The oxygenators used were either the sterile disposable bubble oxygenator of Rygg-Kyvsgaard or the Temptrol oxygenator. All patients were given prophylactic antibiotic cover, which during the early period of the study consisted of 4 million units of penicillin and $1 \mathrm{~g}$ streptomycin every 24 hours from the eve of the day of operation, while during the later period we used $4 \mathrm{~g}$ cloxacillin (Orbenin) and $1 \mathrm{~g}$ kanamycin every 24 hours from the eve of the day of operation.

\section{RESULTS}

The number of cultures started in this way totalled 657 , of which $16(2.4 \%)$ proved to be positive ; all the others (641) remained sterile. Of the 16 patients with a positive blood culture from the heart-lung machine, 12 showed no clinical

\section{T A B LE I}

TOTAL NUMBER OF CULTURES: 657

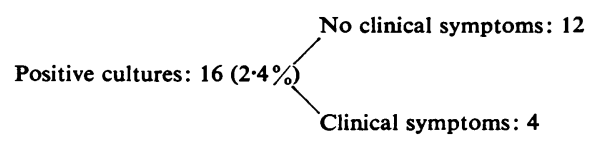

manifestations of infection (Table I), and in these cases the positive finding had no therapeutic consequence. It is quite conceivable that contamination had occurred in these instances. It must be pointed out, however, that one of these 12 patients died immediately after operation. Another patient, who required another operation with extracorporeal circulation after a few weeks, on that occasion had a sterile culture of blood from the heart-lung machine. In several other cases, various subsequent cultures of blood taken under sterile conditions were started as soon as the result of the blood culture from the heart-lung machine was known. All these subsequent cultures remained sterile.

The bacteria cultured from the perfusion blood of these 12 patients are listed in Table II; it should be pointed out that two different micro-

\section{T A B LE II}

BACTERIA CULTURED FROM 12 PATIENTS' WITH NO CLINICAL SYMPTOMS

\begin{tabular}{|c|c|c|c|c|c|}
\hline $\begin{array}{l}\text { Staphylococcus albus } \\
\text { Streptococci of the virid } \\
\text { Indifferent streptococci } \\
\text { Diphtheroid rods . . } \\
\text { Gram-positive tods } \\
\text { Achromobacter } \quad . \\
\text { Pseudomonas } \quad . \\
\text { Alkaligenes faecalis } \\
\text { Sporiferous organisms }\end{array}$ & $\begin{array}{l}\ldots \\
\text { ans } \\
\cdots \\
\cdots \\
\cdots \\
\cdots \\
\cdots\end{array}$ & $\begin{array}{l}\ldots \\
\cdots \\
\cdots \\
\cdots \\
\cdots \\
\cdots\end{array}$ & $\begin{array}{l}\ldots \\
\cdots \\
\cdots \\
\cdots \\
\cdots \\
\cdots\end{array}$ & $\begin{array}{l}\cdots \\
\cdots \\
\cdots \\
\cdots \\
\cdots \\
\cdots \\
\cdots\end{array}$ & $\begin{array}{l}2 \\
1 \\
1 \\
1 \\
3 \\
2 \\
2 \\
2 \\
1 \\
1 \\
1\end{array}$ \\
\hline
\end{tabular}

1 The cultures of two patients yielded two different bacteria. (One patient died immediatedly after operation.) 
organisms were found in the blood from two patients. It is a conspicuous fact that Staphylococcus aureus was never identified.

The other four patients with a positive blood culture from the heart-lung machine did show clinical symptoms; and in three of them there was an unequivocal explanation for the infected perfusion blood. The micro-organisms found in the perfusion blood of these four patients are listed in Table III. Their case histories are briefly discussed.

\section{T A B L E I I I}

BACTERIA FOUND IN FOUR PATIENTS WITH CLINICAL SYMPTOMS

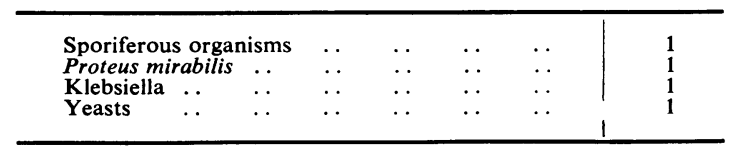

\section{CASE HISTORIES}

CASE 1 Patient F. was a 3-year-old girl in whom a patent ductus arteriosus had been ligated elsewhere three months previously. There was marked postoperative pyrexia, but the temperature returned to normal after one week. Two weeks after the operation, however, acute pyrexia recurred with general malaise and dullness to percussion over the left hemithorax. The leucocyte count was $14,800 \mathrm{~mm}^{3}$, and the ESR was 70-103 $\mathrm{mm} / \mathrm{hr}$. The chest radiograph revealed some pleural exudate, and a round swelling was later demonstrated at the site of the aortic arch. Haemolytic Staph. aureus was repeatedly cultured from blood samples obtained under sterile conditions. With sensitivity as a guideline, the patient was first given chloramphenicol (Globenicol) and subsequently received cloxacillin (Orbenin) and probenecid. When a systolic murmur, which extended into diastole, developed angiocardiography was carried out and disclosed an aneurysm at the site of the ligated ductus arteriosus.

A second operation was then performed with extracorporeal circulation to close the defect in the aorta at the site of the ligated ductus arteriosus. The sutures of the first operation were removed and cultured, yielding a growth of Staph. aureus. Culture of the blood from the heart-lung machine remained sterile. Postoperatively she remained apyrexial, but severe haemothorax developed, with total displacement of the left lung. In view of this it was decided one week later to re-operate with extracorporeal circulation, because we assumed that suture leakage had occurred. The operation disclosed that no new aneurysm had developed and that the aortic suture was adequate. Proteus mirabilis was grown from the clots removed as well as from the perfusion blood. The postoperative course was uneventful apart from a marked wound infection, from which $P$. mirabilis was likewise cultured. The temperature remained normal; the patient received cloxacillin medication for a long time until she had made a complete recovery.

CASE 2 Patient C. was a 57-year-old man who, some 18 months previously, had been treated for a severely calcified aortic stenosis by insertion of a Starr valve. The postoperative period was beset by many complications. The patient was anuric for some time but required no dialysis. Severe bronchitis occurred, and dehiscence of the sternum necessitated re-suturing. Next, persistent anaemia developed which was probably a result of mechanical haemolysis, although insufficiency of the valve was not demonstrable. The patient was treated by multiple blood transfusions and, during long periods, received large doses of various antibiotics. In view of residual inflammatory symptoms, the sternum was re-explored.

Finally, unmistakable insufficiency of the Starr valve occurred, and a re-operation was performed with extracorporeal circulation, even though renal function was still poor (serum creatinine $3.0 \mathrm{mg} /$ $100 \mathrm{ml}$ ). Part of the valve ring had loosened and was resutured. Yeasts were cultured from the perfusion blood. His postoperative temperature was initially normal but after a week began to rise. Blood cultures of samples taken under sterile conditions were repeatedly found to contain yeasts. Micturition was normal during the initial postoperative period but gradually diminished until anuria developed. Dialysis was started but the patient died in toxic anuria on the twelfth postoperative day.

CASE 3 Patient E. was a man aged 50 who, after insertion of a Starr valve into the aorta, developed severe wound infection and mediastinitis, as well as dehiscence of the sternum. Culture of the blood from the heartlung machine remained sterile, but Klebsiella was cultured from the wound. The sternum was resutured when the wound infection had been arrested by conservative measures. Owing to strong adhesions between the anterior wall of the right ventricle and the sternum, a defect in the right ventricle occurred and a repair was undertaken with extracorporeal circulation. Klebsiella was then cultured also from the perfusion blood. With sensitivity as a guideline, the patient was given long-term postoperative treatment with antibiotics. The postoperative course was initially favourable but serious toxic symptoms recurred after about a month. Blood cultures remained sterile but the patient finally died in toxic anuria six weeks after the second operation.

CASE 4 Patient $Z$. was a man of 47 in whom a valvulop!asty of the tricuspid valve was performed with extracorporeal circulation for tricuspid insufficiency, while at the same time a patent foramen ovale was closed. Sporiferous organisms were cultured from the perfusion blood. The postoperative tempera- 
ture was markedly raised, and unmistakable symptoms of sepsis occurred. Sporiferous organisms were likewise cultured from blood samples taken under sterile conditions. Marked wound infection developed followed by severe right-sided empyema. At a second operation under antibiotic cover, the empyema was drained. Both sporiferous organisms and Paracolobactrum were cultured from the thorax. Guided by sensitivity, the patient was given protracted postoperative antibiotic therapy and finally made a complete recovery.

\section{DISCUSSION}

The case histories show that, in three of the four patients with a positive blood culture from the heart-lung machine and unmistakable infective symptoms, a reason could be found for the presence of bacteria in the perfusion blood. These three patients underwent surgery with extracorporeal circulation while an infection was present-an infected aneurysm of the aorta at the site of the ligated ductus arteriosus; an infected Starr valve in the aorta; and mediastinitis. Only in the fourth patient could the infection be ascribed to the extracorporeal circulation.

The incidence of infection as a result of extracorporeal circulation can therefore be described as very low, in spite of the complexity of this procedure which might be expected to entail a high risk of infection. Baffes et al. (1970) believe that this might be explained by dilution of the perfusion fluid in combination with the prophylactic antibiotics. This probably also explains why the other 12 patients with a positive blood culture from the heart-lung machine failed to show any clinical manifestation of infection.

Ankeney and Parker (1969) reported a much higher frequency of positive blood cultures after operations using the heart-lung machine $(19.2 \%$ of 1,555 cultures from 383 patients). But in their series, too, the number of patients with inflammatory symptoms was much smaller than the number of patients with a positive blood culture from the heart-lung machine.

\section{REFERENCES}

Ankeney, J. L. and Parker, R. F. (1969). Staphylococcal endocarditis following open heart surgery related to positive intraoperative blood cultures. In Prosthetic Heart Valves, edited by L. A. Brewer, pp. 719-730. Thomas, Springfield, Illinois.

Baffes, T. G., Blazek, W. V., Fridman, J. L., Augustsson, M. H., and J. van Elk (1970). Postoperative infections in 1,136 consecutive cardiac operations. Surgery, 68, 791-799. 